\title{
Antibiotics Susceptibility Profile and Synergistic Effects of Flavonoids with Antibiotics against Resistant Staphylococcus aureus from Asymptomatic Individuals
}

\author{
Rudra Mishra and Pasupathi Rathinasabapathi* (D) \\ Department of Genetic Engineering, SRM Institute of Science and Technology, Kattankulathur -603203, \\ Tamil Nadu, India.
}

\begin{abstract}
Staphylococcus aureus exhibits resistance to most of the commonly used antibiotics. Although antibiotics suceptibility studies have been performed on the pathogen isolated from the patient samples, only limited information is available about that of $S$. aureus isolated from asymptomatic individuals. In this study, $S$. aureus was isolated from the skin microbiota of the asymptomatic individuals, and susceptibility of the pathogen against different antibiotics and plant flavonoids was compared to drug-sensitive strain. The minimum inhibitory concentration (MIC) value and IC50 of the pathogen were calculated against the antibiotics and flavonoids. The susceptibility pattern of the isolated strain showed higher resistance against erythromycin $\left(100 \mu \mathrm{g} \mathrm{m}^{-1}\right)$ and vancomycin $(25 \mu \mathrm{g}$ $\mathrm{mL}^{-1}$ ). Based on the fractional inhibitory concentration index (FICl) values, the combinatory effects of antibiotics and flavonoids were categorized into synergistic, additive, and indifferent. The combination of rutin and erythromycin showed a synergistic effect with the concentrations of $31.25 \mu \mathrm{g} \mathrm{mL}^{-1}$ and 1.562 $\mu \mathrm{g} \mathrm{mL}^{-1}$ against drug-sensitive strains of $S$. aureus. Similarly, the same combination showed synergistic effects against isolated strains at the concentration of $625 \mu \mathrm{g} \mathrm{m}^{-1}$ and $12.5 \mu \mathrm{g} \mathrm{mL}^{-1}$. We observed an increase in drug resistance in the isolated strain of $S$. aureus in comparison to the drug-sensitive strain. To the best of our knowledge, this was the first study reporting increase in antimicrobial resistance of $S$. aureus present on asymptomatic individuals than the sensitive strain.
\end{abstract}

Keywords: Asymptomatic, S. aureus, Synergistic effects

*Correspondence: rathinap1@srmist.edu.in; +91 9790720198

(Received: October 14, 2020; accepted: November 26, 2020)

Citation: Mishra R, Rathinasabapathi P. Antibiotics Susceptibility Profile and Synergistic Effects of Flavonoids with Antibiotics against Resistant Staphylococcus aureus from Asymptomatic Individuals. J Pure Appl Microbiol. 2020;14(4):2669-2676. doi: 10.22207/JPAM.14.4.44

(C) The Author(s) 2020. Open Access. This article is distributed under the terms of the Creative Commons Attribution 4.0 International License which permits unrestricted use, sharing, distribution, and reproduction in any medium, provided you give appropriate credit to the original author(s) and the source, provide a link to the Creative Commons license, and indicate if changes were made. 


\section{INTRODUCTION}

Staphylococcus aureus is a non-motile, non-spore-forming gram-positive microorganism that produces catalase and coagulase enzymes ${ }^{1-3}$. Although S. aureus is typically a commensal organism, it is also known opportunistic organism. S. aureus is a human commensal that persistently colonizes the anterior nares and skin of $20 \%-25 \%$ of the world population $n^{4-6}$. S. aureus causes numerous diseases, including scalded skin syndrome, abscesses, septicemia, pneumonia, food poisoning, and toxic shock syndrome through invasive wound infections ${ }^{7-8}$. S. aureus is also one of the significant hospital-acquired pathogens causing post-operative infections, toxic shock syndrome, food poisoning, osteomyelitis, and endocarditis ${ }^{9}$.

The abuses in drug usage lead to the acquired antimicrobial resistance to many pathogens, including $S$. aureus. The biofilm-ability of $S$. aureus during the infection increases its tolerance against most of the available antimicrobials ${ }^{10-11}$. The biofilm prevents phagocytosis; thus establishing chronic infections ${ }^{12}$. The skin microbiota of healthy individuals harbors a significant amount of $S$. aureus. A recent study showed that non-clinical isolates of $S$. aureus exhibited antimicrobial resistance ${ }^{13-14}$. The carriage of drug-resistant strains in healthy individuals becomes a major asymptomatic reservoir, which can rapidly spread infection within the community ${ }^{15}$.

As all the commercially available drugs are ineffective in treating the drug-resistance pathogens, alternative approaches for effective treatments should be developed. The combination of drug treatments with flavonoids and conventional antibiotics could control the multidrug-resistant pathogens more effectively ${ }^{16-18}$. Flavonoids are secondary metabolites produced in different parts of the plant, and it exhibit antimicrobial activity against a broad range of pathogens. Also, flavonoid based treatments are effective in controlling the biofilm formation in antibiotics resistant $S$. aureus. The plant metabolites reinforce the antibiotics to enhance their efficacy in the combination treatments ${ }^{19}$. The naringenin and sophora $G$ inhibit the multidrug-resistant S. aureus (MRSA) by reducing the fluidity of the cellular membranes ${ }^{20}$. Quercetin, rutin, and salidroside disturb the cellular membrane's integrity and biofilm formation of various pathogens ${ }^{21}$. Quercetin and antibiotics exhibited superior inhibitory effects on $S$. aureus. In these combinatory treatments, the flavonoids increase the cell permeability, increasing the uptake of the antibiotic by the pathogen ${ }^{22}$.

The earlier studies had reported the effect of combinatory treatments on the clinically isolated strains of $S$. aureus and MRSA. In this study, we have compared the drug susceptibility patterns between drug-sensitive strains of $S$. aureus and isolated strains from healthy individuals. Further, the study evaluated the synergistic effects of different flavonoids with antibiotics against both strains.

\section{MATERIALS AND METHODS \\ Antimicrobials, Media, and Culture}

All the antibiotics (erythromycin, vancomycin, tetracycline, rifampicin, and gentamicin), and flavonoids (quercetin, rutin, morin, and naringenin) were purchased from Himedia Laboratories Pvt. Ltd., Mumbai, India. The stock solution of antibiotics and flavonoids were prepared in the respective solvents. The drugsensitive strain S. aureus (MTCC96) was purchased from Microbial Type Culture Collection and Gene Bank (MTCC), Chandigarh, India. The bacterial strain was cultured on mannitol salt agar (MSA). The Mueller-Hinton broth was used to determine the susceptibility analysis and synergistic effects using checkerboard assay. All the media were procured from Himedia Laboratories, India.

Isolation of Staphylococcus aureusf rom asymptomatic individuals

A total of 15 healthy volunteers were chosen for the isolation of $S$. aureus from their skin. S. aureus cultured in the MSA, which is the selective and differential medium for isolating and identifying $S$. aureus. The MSA contains $1 \%$ mannitol, $7.5 \%$ sodium chloride, phenol red indicator, and peptone. $S$. aureus can tolerate and survive in the high salt condition in this medium. In contrast, other bacteria's growth is inhibited owing to the high salt concentration of the media. S. aureus can produce the acid end product by fermenting mannitol sugar in the media, which changes the phenol red indicator into yellow. In this experiment, the suspected single bacterial colony was inoculated on an MSA plate (Oxoid, 
Basingstoke, UK) incubated at $35^{\circ} \mathrm{C}$ for $24 \mathrm{~h}$, and colour change of the indicator was observed. Furthermore, the genomic DNA was isolated from the selected $S$. aureus, and the 16s rRNA gene sequencing method was used for the species confirmation.

\section{Antimicrobial susceptibility of $S$. aureus}

The susceptibility of drug-sensitive and clinical isolates against antimicrobials (antibiotics and flavonoids) was compared using the broth micro-dilution method as per the clinical and laboratory standard institute (CLSI) guidelines ${ }^{23-24}$. The stock solutions of the antibiotics $\left(100 \mu \mathrm{gmL}^{-1}\right.$. $0.78 \mu \mathrm{g} \mathrm{mL}^{-1}$ ) and flavonoids ( $2 \mathrm{mg} \mathrm{mL}^{-1}-0.015 \mathrm{mg}$ $\left.\mathrm{mL}^{-1}\right)$ were prepared in the appropriate solvents. The bacterial suspensions with $5 \times 10^{8}$ colony forming units (CFU mL $\mathrm{m}^{-1}$ ) concentration were prepared by diluting the bacteria in Muller Hinton broth (MHB) and added into the 96-well plate. The bacterial suspension in each row was added with a varying dilution of specific antimicrobials The antimicrobials were added upto eight fold dilution from the stock concentration. Then, the cultures were incubated at $37^{\circ} \mathrm{C}$ for $24 \mathrm{~h}$. After the incubation, $20 \mu \mathrm{L}$ of resazurin dye with concentration of $0.15 \mathrm{mg} \mathrm{mL}^{-1}$ was added to each well, and incubated at $37^{\circ} \mathrm{C}$ for $4 \mathrm{~h}$. The pink color indicated the live cells, whereas the blue color indicated dead cells. The color change was used to estimate the bacterial growth of the pathogen. For fluorescence-based detection, fluorescence with excitation and emission at $530 \mathrm{~nm} / 590 \mathrm{~nm}$ was measured $^{25}$. All the experiments were performed in triplicate.

\section{Synergistic effects of flavonoids and antibiotics on planktonic cells}

The synergistic effects of a different combination of flavonoids and antibiotics were evaluated using the checkerboard assay study ${ }^{26}$. The 64 different concentrations of antibiotics and flavonoids ranged between $4 \mathrm{X}$ MICand $1 / 32 X$ MIC values were prepared in MHB broth and tested for it combinatory inhibitory effects. Briefly, the $S$. aureus was added to the plate at a final concentration of $1.5 \times 10^{8}$ colony forming units (CFU mL ${ }^{-1}$ ). The different concentration of anitbiotics (1/32X $-4 \mathrm{X}$ MIC) were added at each column of the 96 -well plate (column III to column $\mathrm{X})$. Similarly, different concentration of flavonoids were added in each row (row to row VIII). The $\mathrm{MHB}$ broth was used as the negative control,and bacterial culture without antibiotics or flavonoids was used as positive control. After incubating at $37^{\circ} \mathrm{C}$ for $24 \mathrm{~h}, 20 \mu \mathrm{L}$ of the resazurin dye $(0.15 \mathrm{mg}$ $\mathrm{mL}^{-1}$ ) was added to each well. The plate was again incubated at $37^{\circ} \mathrm{C}$ for $4 \mathrm{~h}$. The fluorescence absorbance of the dye was measured at excitation $(530 \mathrm{~nm})$ and emission $(590 \mathrm{~nm})$. The change of color from dark blue to pink is indicated the metabolic activeness of the culture. As described above, the MIC values of antibiotics and flavonoids in the combinations were evaluated using the above resazurin dye-based method.

The synergistic effect of the drug combinations was tested by calculating the Fractional inhibitory concentration index $(\mathrm{FICl})$ as mentioned below: $\mathrm{FICl}_{\mathrm{A}+\mathrm{B}}=\mathrm{FIC}_{\mathrm{A}}+\mathrm{FIC}_{\mathrm{B}}$, where $\mathrm{FIC}_{\mathrm{A}}$ $=\mathrm{MIC}_{\mathrm{A}}^{\text {combi }} / \mathrm{MIC}_{\mathrm{A}}$ alone and $\mathrm{FIC}_{\mathrm{B}}=\mathrm{MIC}_{\mathrm{B}}^{\text {combi } / \mathrm{MIC}_{\mathrm{B}} \text { alone }}$.

Table 1. IC50 value (Inhibitory Concentration) and Minimum Inhibitory Concentration (MIC) value of antibiotics and flavonoids against Staphylococcus aureus

\begin{tabular}{|c|c|c|c|c|}
\hline \multirow{2}{*}{$\begin{array}{l}\text { Antibiotics / } \\
\text { Flavonoids }\end{array}$} & \multicolumn{2}{|c|}{ MIC Value } & \multicolumn{2}{|c|}{ IC50 value } \\
\hline & $\begin{array}{l}\text { MTCC strain } \\
\left(\mu \mathrm{g} \mathrm{mL} \mathrm{m}^{-1}\right)\end{array}$ & $\begin{array}{l}\text { Isolated strain } \\
\quad\left(\mu \mathrm{gL} \mathrm{m}^{-1}\right)\end{array}$ & $\begin{array}{c}\text { MTCC strain } \\
\left(\mu \mathrm{g} \mathrm{mL}^{-1}\right)\end{array}$ & $\begin{array}{l}\text { Isolated strain } \\
\quad\left(\mu \mathrm{gL} \mathrm{L}^{-1}\right)\end{array}$ \\
\hline Erythromycin & 6.25 & 100 & 2.628 & 43.62 \\
\hline Vancomycin & 6.25 & 25 & 2.549 & 16.20 \\
\hline Rifampicin & 0.78 & 3.125 & 0.7615 & 1.878 \\
\hline Gentamicin & 0.78 & 1.56 & 0.7763 & 2.068 \\
\hline Tetracycline & 0.78 & 3.125 & 0.755 & 1.473 \\
\hline Quercetin & 250 & 625 & 118.2 & 366.2 \\
\hline Rutin & 250 & 2500 & 127.9 & 751 \\
\hline Morin & 125 & 1250 & 73.62 & 700 \\
\hline Naringenin & 625 & 625 & 481.4 & 591.5 \\
\hline
\end{tabular}


The $\mathrm{MIC}_{\mathrm{A}}{ }^{\mathrm{combi}}$ and $\mathrm{MIC}_{B}{ }^{\text {combi }}$ are the MIC values of drug $A$ in combination, while $\mathrm{MIC}_{A}$ alone and $\mathrm{MIC}_{B}$ alone are the individual MIC values. The effect of the drug combinations was catagorized into different groups based on the $\mathrm{FICl}$ values (Table 2): $\mathrm{FICl} \leq 0.5$ showed the synergistic effect; $\mathrm{FICl}$ range between $>0.5$ and $\leq 1.0$ showed the additive or partial effect; $\mathrm{FICl}$ value range between $>1.0$ and $\leq 4.0$ showed the indifferent effect, and FIC $>4$ showed the antagonistic effect ${ }^{27-28}$.

\section{Statistical analysis}

Drug dose-response inhibition curve and Isobologram were plotted and analyzed using GraphPad Prism version 8.0 for Windows, GraphPad Software, La Jolla California USA. The $p$ values of $\leq 0.05$ were considered as significantly different. Similarly, the IC50 value was calculated, and graphs were plotted for the percentage of inhibition using the fluorescence Intensity. $\mathrm{FICl}$ values were calculated using the Loeweadditivity zero-interaction theory. The strength of a relationship based on its $\mathrm{R}$-squared value: if $R$-squared value $<0.3$, it is considered as none or very weak effect size, $0.3<r<0.5$ value was considered a weak or low effect size, $0.5<r<0.7$ value was considered as oderate effect size, and $r>$ 0.7 value was considered as strong effect size ${ }^{29}$. All data were presented as mean values with standard deviations.

\section{RESULTS AND DISCUSSIONS Isolation of $S$ aureus from asymptomatic individuals}

The isolated strains of $S$. aureus from the skin microbiota of asymptomatic individuals were grown in MSA medium. Mannitol fermentation produces an acid, which is detected by phenol red $\mathrm{pH}$ indicator. Since the Staphylococcus aureus is mannitol fermenting organism, it produces yellow-colored colonies in the MSA medium, while the other Staphylococcus species are unable to ferment mannitol. Furthermore, the DNA sequencing of the $16 \mathrm{~s}$ rRNA gene was confirmed the $S$. aureus.

Antimicrobial Susceptibility profiling of $S$. aureus The broth dilution method was used to determine the MIC of four flavonoids and five antibiotics against planktonic cells of $S$. aureus of drug-sensitive (MTCC96) and non-clinical isolated strain. The plant flavonoids quercetin, rutin and morin have been found to posses the antibactericidal activity The MIC values were determined for the aforementioned antimicrobial individually and in combination. The individual MIC values showed that the non-clinical isolates were more resistant than the drug-sensitive strain. The non-clinical isolated strain had 16 -fold higher MIC values $\left(100 \mu \mathrm{g} \mathrm{mL} \mathrm{m}^{-1}\right)$ for erythromycin than the drug-sensitive strain $\left(6.25 \mu \mathrm{g} \mathrm{mL}^{-1}\right)$. Also,other antibiotics had a two - four fold increase in MIC

Table 2. Combinatory inhibitory effects of flavonoids with antibiotics against Staphylococcus aureus

\begin{tabular}{|c|c|c|c|c|c|c|c|}
\hline \multicolumn{2}{|c|}{$\begin{array}{l}\text { Antibiotics } \\
\text { /Flavonoids }\end{array}$} & \multicolumn{3}{|c|}{ MTCC 96} & \multicolumn{3}{|c|}{ Isolated strain } \\
\hline & & $\begin{array}{c}\text { MIC in } \\
\text { combinations } \\
\left(\mu \mathrm{g} \mathrm{mL}^{-1}\right)\end{array}$ & $\mathrm{FICl}$ & Effect & $\begin{array}{c}\text { MIC in } \\
\text { combinations } \\
\left(\mu \mathrm{g} \mathrm{mL}^{-1}\right)\end{array}$ & $\mathrm{FICl}$ & Effect \\
\hline \multirow[t]{2}{*}{ Que } & Ery & $125 / 3.125$ & 1.00 & Indifferent & 156.2 / 6.25 & 0.26 & Synergistic \\
\hline & Van & 250 / 0.390 & 1.06 & Indifferent & $19.5 / 25$ & 1.03 & Indifferent \\
\hline \multirow[t]{2}{*}{ Mor } & Ery & 62.5 / 0.781 & 0.62 & Additive & $625 / 25$ & 0.75 & Additive \\
\hline & Van & $31.25 / 0.390$ & 0.31 & Synergistic & $78 / 12.5$ & 0.56 & Additive \\
\hline \multirow[t]{2}{*}{ Rut } & Ery & 31.25 / 1.562 & 0.38 & Synergistic & $625 / 12.5$ & 0.38 & Synergistic \\
\hline & Van & 125 / 0.781 & 1.13 & Indifferent & 156.2 / 12.5 & 0.56 & Additive \\
\hline \multirow[t]{2}{*}{ Nar } & Ery & 312.5 / 1.562 & 0.75 & Additive & $156.2 / 25$ & 0.5 & Synergistic \\
\hline & Van & 312.5 / 0.195 & 0.53 & Synergistic & $78 / 12.5$ & 0.62 & Additive \\
\hline
\end{tabular}

Note : FICl - Fractional Inhibitory Concentration Index, MIC - Minimum Inhibitory Concentration, Que - Quercetin, Rut - Rutin, Mor - Morin, Nar - Naringenin, Ery - Erythromycin, Van - Vancomycin 
for the non-clinical isolates (Table 1). Even plant flavonoids showed a similar increase in MIC values for the non-clinical isolates. Rutin and morin had a 10-fold increase in MIC values, with rutin posses the highest MIC among the other flavonoids (Table 1). Only naringenin showed equal MIC for both strains. The drug response effect of each antibiotics and flavonoids was measured at different concentrations to calculate antimicrobial concentration to the achieve the $50 \%$ of maximal inhibition (IC50) 30 Fig. 1). Even the IC50 values showed that the concentrations of antibiotics required to inhibit the growth of the isolated strain of $S$. aureus are higher (Table 1).

Most of the population is colonized asymptomatically by $S$. aureus in the regions of nares, skin, and rectal areas ${ }^{31}$. Carriage of $S$. aureus rarely causes disease in healthy individuals, but it is associated with an increased risk for the emergence of infections in various populations ${ }^{32-34}$. A previous report on antimicrobial susceptibility pattern of $S$. aureus isolated from the nosal carriage in preschool childrens showed resistance to $\beta$-lactum antibiotics $^{35}$. A study compared the antimicrobial resistance of $S$. aureus from nasal carriage and clinical samples. The clinical isolates exhibited higher resistance for aminoglycosides, while carrier isolates had higher resistances against quinolones and tetracycline ${ }^{36}$. However, no reported study to compare the susceptibility pattern between the skin microbiota and drug-sensitvitive strain. Also, this study reported an increase in resistance to non-clinical isolates of $S$. aureus against both antibiotics and plant flavonoids, which is alarming. The combinatory treatement would be a more appropriate method in controlling the acquired antimicriobial resistnance.

\section{Synergistic effect of flavonoids in combination with antibiotics on planktonic cells}

A drug combination is called synergistic when the combined drug treatment provides superior effects than individual drug effects. In this study, we have evaluated the synergistic effects of four flavonoids (quercetin, rutin, morin, and naringenin) with antibiotics (erythromycin and vancomycin) on planktonic cells of $S$. aureus by the checkerboard assay. As the non-clinical isolated strains exhibited more resistance against vancomycin and erythromycin, therefore these antibiotics were used for combinatory drug treatments. The synergistic effects were observed in the quercetin, rutin, naringenin in combination with erythromycin in non-clinical isolates with $\mathrm{FICl}$ values rangeing between 0.26 and 0.5 . Against drug-sensitive strain, the synergistic effect was observed in the combination of antibiotic vancomycin with the plant flavonoids of naringenin, morin, and erythromycin and rutin. The $\mathrm{FICl}$ values of the above combinations ranged from 0.31 to 0.5 (Table 2). The study found that
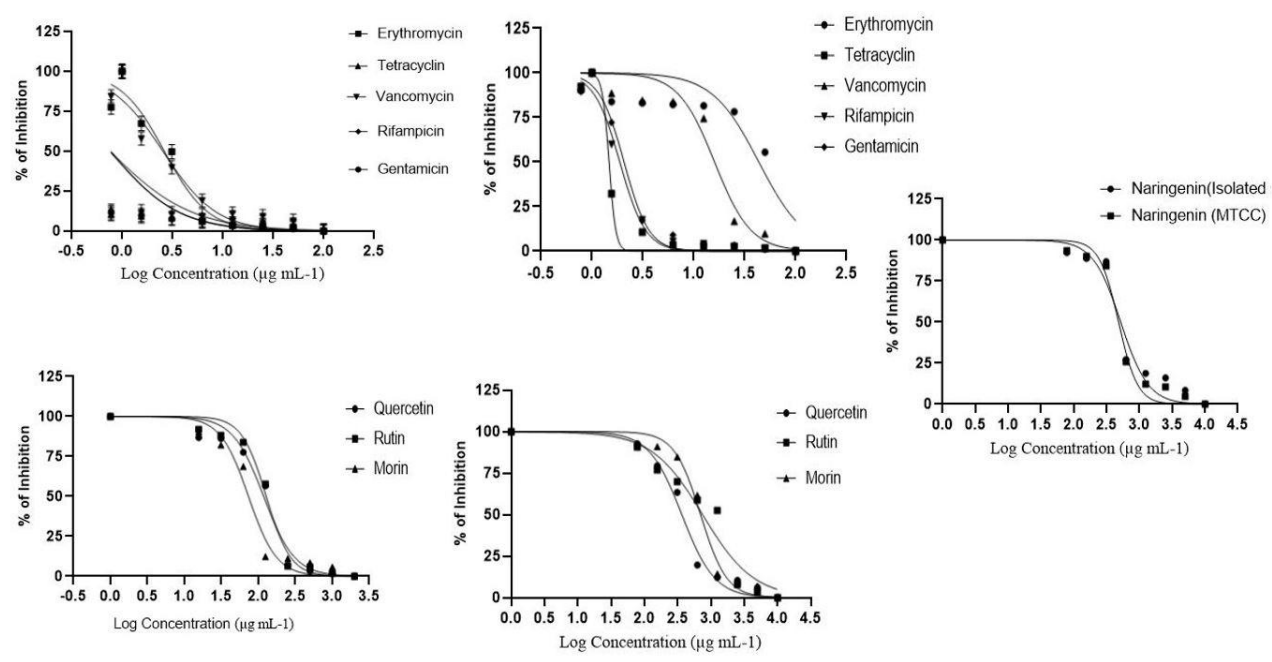

Fig. 1. Effect of antibiotics and flavonoids against the planktonic cells of S. aureus (Dose-response - inhibition curve). A - MTCC strain and B - Isolated strain for antibiotics, C - MTCC strain and D - Isolated strain for flavonoids, and E - Naringenin for both strain. 
erythromycin had a high level of synergistic effects with most flavonoids than the other antibiotics. The indifferent effect was found in vancomycin and quercetin combination against non-clinical isolated strains $S$. aureus, and three combinations against drug-sensitive strain with the $\mathrm{FICl}$ values of one. Four drug combinations showed the additive effects in the non-clinical isolates of $S$. aureus. The quercetin and rutin reduced 16 -fold and eight fold MIC of erythromycin, respectively. The morin and naringenin reduced four fold of erythromycin . The MIC of vancomycin reduced to one -two fold with all the flavonoids (Table 1 and Table 2). Many studies reported the treatment combination against MRSA, not against the nonclinical isolates. Against MRSA, quercetin showed synergistic effects with the antibiotics and decreased four fold MIC of antibiotics. The morin and rutin had indifferent effects with antibiotics ${ }^{37}$. A earlier study reported that quercetin and morin decreased 3-16 fold MIC of tertracyclin, ciprofloxacin and erythromycin against $S$. aureus ${ }^{38}$, which is similar to this study. The results of this study is similar to the abovementioned reports on combinatory treatments. Therefore, the addition of flavonoids in an antimicrobial treatment against $S$. aureus reduces the required antibiotics dosage, which aid in controlling the acquired resistance of the pathogen.

\section{CONCLUSIONS}

S. aureus forms the colonizes in the nasal passage and axillae of humans. The suceptability pattern against antibiotics and flavonoids was evaluated for $S$. aureus from the asymptomatic individuals. The MIC and IC50 values indicated an increase in resistance in non-clinical isolates in comparision with drug-sensitive against all the antibiotics and flavonoids. The 16-fold increased MIC values of erythromycin and two-four fold MIC of other antibiotics was observed for nonclinical isolates. Except naringenin, all other flavonoids had increased MIC values for isolated strain. In non-clinical isolates, the synergistic effects were found between erythromycin with most of the flavonoids, whereas the vancomycin shows only the additive effects with flavonoids. The combinatory treatments with flavonoids reduced the the required MIC of antibiotics upto 4-16-fold in combination than that of individual treatment .The study shows that the combinatory treatment of flavonoids are an effective approach to overcome bacterial resistance.

\section{ACKNOWLEDGMENTS}

We would like to acknowledge the SRM Institute of Science and Technology, TamilNadu, India, for their financial support and facilities to carry out this project. We would also like to thank Osheen Sharma, Department of Genetic Engineering, SRM Institute of Science and Technology for doing statistical analysis and data interpretation.

\section{CONFLICT OF INTEREST}

The authors declare that there is no conflict of interest.

\section{AUTHORS' CONTRIBUTION}

All authors designed the experiments. RM performed the experiments. RM and $P R$ analyzed the data. RM drafted the manuscript. RP supervised the revised the manuscript. All the authors read and approved the manuscript.

\section{FUNDING}

None.

\section{DATA AVAILABILITY}

All datasets generated or analyzed during this study are included in the manuscript.

\section{ETHICS STATEMENT}

Not applicable.

\section{REFERENCES}

1. O'Riordan K, Lee JC. Staphylococcus aureus capsular polysaccharides. Clin Microbiol Rev. 2004;17(1):218234. doi: $10.1128 / \mathrm{cmr}$.17.1.218-234.2004

2. Ho J, O'Donoghue MM, Boost MV. Occupational exposure to raw meat: a newly-recognized risk factor for Staphylococcus aureus nasal colonization amongst food handlers. Int J Hyg Environ Health. 2014;217(23):347-353. doi: 10.1016/j.ijheh.2013.07.009

3. Williams RE. Healthy carriage of Staphylococcus aureus: its prevalence and importance. Microbiol Mol Biol Rev. 1963;27(1):56-71. doi: 10.1128/ MMBR.27.1.56-71.1963

4. Eriksen NH, Espersen F, Rosdahl VT, Jensen K. Carriage of Staphylococcus aureus among 104 healthy persons during a 19-month period. Epidemiol Infect. 1995;115(1):51-60. doi: 10.1017/s0950268800058118

5. $\mathrm{Hu}$ L, Umeda A, Kondo S, Amako K. Typing of 
Staphylococcus aureus colonising human nasal carriers by pulsed-field gel electrophoresis. J Med Microbiol. 1995;42(2):127-132. doi: 10.1099/00222615-42-2-127

6. Kluytmans J, van Belkum A, Verbrugh H. Nasal carriage of Staphylococcus aureus: epidemiology, underlying mechanisms, and associated risks. Clin Microbiol Rev. 1997;10(3):505-520. doi: 10.1128/CMR.10.3.505520.1997

7. Moore PCL, Lindsay JA. Genetic variation among hospital isolates of methicillin-sensitive Staphylococcus aureus: Evidence for horizontal transfer of virulence genes. J Clin Microbiol. 2001;39(8):2760-2767. doi: 10.1128/JCM.39.8.2760-2767.2001

8. Boyd EF, Brussow H. Common themes among bacteriophage-encoded virulence factors and diversity among the bacteriophages involved. Trends Microbiol. 2002;10(11):521-529. doi: 10.1016/S0966842X(02)02459-9

9. Benayache $S$, Benayache $F$, Benyahia $S$, et al. Leaf oils of some Eucalyptus species growing in Algeria. J Essent Oil Res. 2001;13:210-213. doi: 10.1080/10412905.2001.9699668

10. Costerton JM, Donlan JM. Biofilms: Survival Mechanisms of Clinically Relevant Microorganisms. Clin Microbiol Rev. 2002;15(2):167-193. doi: 10.1128/ cmr.15.2.167-193.2002

11. Bjarnsholt T, Fazli M, Kirketerp-Moller K, et al. Nonrandom Distribution of Pseudomonas aeruginosa and Staphylococcus aureus in Chronic Wounds. J Clin Microbiol. 2009;47(12):4084-4089. doi: 10.1128/ JCM.01395-09

12. Hoiby N, Bjarnsholt T, Michael T, Molin G, Ciofu $O$. Antibiotic resistance of bacterial biofilms. Int $J$ Antimicrob Agents. 2010; 35(4): 322-332. doi: 10.1016/j.ijantimicag.2009.12.011

13. Cogen AL, Nizet V, Gallo RL. Skin Microbiota: A Source of Disease or Defence? Br J Dermatol. 2008;158(3):442455. doi: 10.1111/j.1365-2133.2008.08437

14. Otto M. Physical stress and bacterial colonization. FEMS Microbiol Rev. 2014;38(6):1250-1270. doi: 10.1111/1574-6976.12088

15. Davoodabadi F, Mobasherizadeh S, Mostafavizadeh K, et al. Nasal colonization in children with community acquired methicillin-resistant Staphylococcus aureus. Adv Biomed Res. 2016;5(86). doi: 10.4103/22779175.182217

16. Sakagami $Y$, linuma $M$, Piyasena KGNP, Dharmaratnec HRW. Antibacterial activity of a-mangostin against vancomycin resistant Enterococci (VRE) and synergism with antibiotics. Phytomedicine. 2005;12(3):203-208. doi: 10.1016/j.phymed.2003.09.012

17. Wang $Y$, Inui $T$, Deng $S$, Smithc DC, Franzblaua SG, Pauliab GF. Counter-current chromatography based analysis of synergy in an anti-tuberculosis ethnobotanical. J Chromatogr A. 2007;1151(1-2):211215. doi: 10.1016/j.chroma.2007.01.127

18. Phitaktim $S$, Chomnawang $M$, Sirichaiwetchakoon K, Dunkhunthod B, Hobbs G, Eumkeb G. Synergism and the mechanism of action of the combination of a-mangostin isolated from Garcinia mangostana $\mathrm{L}$. and oxacillin against an oxacillin-resistant Staphylococcus saprophyticus. BMC Microbiol. 2016;16:195. doi:

\subsection{6/s12866-016-0814-4}

19. Sana M, Jameel H, Rahman M. Miracle remedy: inhibition of bacterial efflux pumps by natural products. J Infect Dis Ther. 2015;3(2):1000213. doi: 10.4172/2332-0877.1000213

20. Tsuchiya $\mathrm{H}$, linuma M. Reduction of Membrane Fluidity by Antibacterial Sophoraflavanone $\mathrm{G}$ Isolated from Sophora exigua. Phytomedicine. 2000;7(2):161-165. doi: 10.1016/S0944-7113(00)80089-6

21. Sanver D, Murray BS, Sadeghpour A, Rappolt M, Nelson AL. Experimental modeling of flavonoid-biomembrane interactions. Langmuir. 2016;32:13234-13243. doi: 10.1021/acs.langmuir.6b02219

22. Stepanovic S, Dakic I, Opavski N, et al. Influence of the growth medium composition on biofilm formation by Staphylococcus sciuri. Annals Microbiol. 2003;53:6374.

23. CLSI (Clinical and Laboratory Standards Institute). Performance standards for antimicrobial susceptibility testing. 23th informational supplement. CLSI document M100-S25. 2015.

24. Aradhana AV, Srividya ND, Roy $P$, et al. Antistaphylococcal activity of bacteriophage derived chimeric protein P128. BMC Microbiol. 2012;12(41):19. doi: 10.1186/1471-2180-12-41

25. Goughenour KD, Balada-Llasat J-M, Rappleyea CA. Quantitative microplate-Based Growth Assay for determination of antifungal susceptibility of Histoplasma capsulatum yeasts. I Clin Microbiol. 2015;53(10):3286-3295. doi: 10.1128/JCM.00795-15

26. Schwalbe R, Steele-Moore L, Goodwin AC. Antimicrobial susceptibility testing protocols, CRC Press. New York. 2007. doi: $10.1201 / 9781420014495$

27. Odds C. Synergy, antagonism, and what the chequerboard puts between them. J Antimicrob Chemother. 2003;52(1):1. doi: 10.1093/jac/dkg301

28. European Committee for Antimicrobial Susceptibility Testing (EUCAST) of the European Society of Clinical Microbiology and Infectious Dieases (ESCMID). EUCAST Definitive Document E.DEF 3.1, Determination of minimum inhibitory concentrations (MICs) of antibacterial agents by agar dilution. Clin Microbiol Infect. 2000;6(9):509-515. doi: 10.1046/j.14690691.2000.00142.x

29. Moore DS, Notz WI, Flinger MA. The basic practice of statistics, New York, NY: W. H. Freeman and Company. 2013.

30. Zhang $\mathrm{H}$, Holden-Wiltse J, Wang J, Liang H. A strategy to model nonmonotonic dose-response curve and estimate IC50. PloS ONE. 2013;8(8):e69301. doi: 10.1371/journal.pone.0069301

31. Wertheim HF, Melles DC, Vos MC, et al. The role of nasal carriage in Staphylococcus aureus infections. Lancet Infect Dis. 2005;5(12):751-762. doi: 10.1016/ S1473-3099(05)70295-4

32. Cohen J, Powderly WG, Opal SM. Infectious Diseases, 3rd ed. London: Mosby Elsevier Science. 2010.

33. Davis JP, Fox BC. Hospital and clinics community associated methicillin resistant Staphylococcus aureus (CA MRSA): Guidelines for clinical management and control of transmission, University of Wsconsin, 2005. https://www.researchgate.net/ 
publication/237262946_community_associated_ methicillin_resistant_staphylococcus_aureus_CA_ MRSA_Guidelines_for_Clinical_Management_and_ Control_of_Transmission.

34. Fridkin SK, Hageman JC, Morrison M, Sanza et al. Active bacterial core surveillance program of the emerging infections program network. Methicillin-resistant Staphylococcus aureus disease in three communities, N Engl J Med. 2005;352(14):1436-1444.

35. Reta A, Wubie M, Mekuria G. Nasal colonization and antimicrobial susceptibility pattern of Staphylococcus aureus among pre-school children in Ethiopia. BMC Res Notes. 2017;10(1):746. doi: 10.1186/s13104-0173079-6

36. Ayepola OO, Olasupo NA, Egwari LO, Becker K, Schaumburg F. Molecular characterization and antimicrobial susceptibility of Staphylococcus aureus Isolates from clinical infection and asymptomatic carriers in Southwest Nigeria. PLOS ONE. 2015;10(9):e0137531. doi: 10.1371/journal. pone. 0137531

37. Amin, Khurram M, Khattak M, Khan J. Antibiotic additive and synergistic action of rutin, morin and quercetin against methicillin resistant Staphylococcus aureus. BMC complement Alternat Med. 2015;15(59):01-12. doi: 10.1186/s12906-015-0580-0

38. Abreu AC, Serra SC, Borges A, et al. Combinatorial activity of flavonoids with antibiotics Against drugresistant Staphylococcus aureus. Microb Drug Resist. 2015; 21(6):600-609. doi: 10.1089/mdr.2014.0252. PMID: 25734256 